\title{
Hand Hygiene Knowledge, Practices and Attitudes among Nurses and Physicians
}

\author{
Mu'taz M. Dreidi', Intima Alrimawi', Ahmad R. Saifan ${ }^{2 *}$, Abdul-Monim Batiha ${ }^{3}$ \\ ${ }^{1}$ School of Nursing, Birzeit University, Ramallah, Palestine \\ ${ }^{2}$ School of Nursing, Applied Science Private University, Amman, Jordan \\ ${ }^{3}$ School of Nursing, Philadelphia University, Amman, Jordan \\ Email: *a_saifan@asu.edu.jo
}

Received 3 February 2016; accepted 15 March 2016; published 18 March 2016

Copyright (C) 2016 by authors and Scientific Research Publishing Inc.

This work is licensed under the Creative Commons Attribution International License (CC BY). http://creativecommons.org/licenses/by/4.0/

(c) (i) Dpen Access

\begin{abstract}
Background: Hand hygiene is one of the most effective ways to control health care related infection. Nurses and physicians are the main health care workers contacting with patients, representing the vector in the chain of infection. Thus, assessing their knowledge, practice and attitudes regarding hand hygiene is very important to decrease the incidence of health care related infection and to improve quality of care. Aims: The aim is to assess the knowledge, practice and attitude of Palestinian physicians and nurses regarding hand hygiene in hospitals. Methods: The study adopted a cross-sectional, quantitative design. Nurses and doctors who worked in the major governmental and private hospitals in the West Bank (Palestine) were targeted. Data was collected using Hand Hygiene Questionnaire. Results: 200 nurses and physicians participated in this study. The results showed that the participants had a moderate knowledge regarding the hand hygiene $(\mathrm{m}=$ $6, S D=1.7$ ). They had a better attitude score than practice with a mean of $82.5 \pm 8.8$. There was a significant difference between male and female only in practice score $(p=0.015)$. Older participants had better attitudes, and private hospitals had significantly higher scores for compliance, importance of hand hygiene and practice than governmental hospitals $(p<0.05)$. Conclusion: The results of the current study showed that there was a further need to focus on the practices of hand hygiene by continuous education for both Palestinian nurses and physicians.
\end{abstract}

\section{Keywords}

Health Care, Infection, Hand Hygiene, Palestine

\section{Introduction}

Health care related infection (HCRI) in hospitals has resulted in many negative impacts on patients, families and 
healthcare workers worldwide [1]. It represents a major burden around the world and affects the safety and quality of care for patients [2]. WHO ranks HCRI as one of the top ten causes of hospital deaths each year [3].

Patients, health care workers, and the environment are major reservoirs of health care related infection [1] [4]. The transmission of infection from patient to patient mainly occurs at the hand of health care workers [5] [6]. Patients' skin, mucous and any discharge can be colonized by many organisms, which will be transferred to surrounding surfaces and contaminate the environment in the hospital [7]. The hands of the health care workers will be contaminated through daily caring for patient or his environment, despite wearing gloves [8]. Therefore, hand hygiene $(\mathrm{HH})$ is considered the most effective way to prevent cross-transmission of HCRI [6] [9]. Health care related infection is estimated to affect $10 \%$ of patients in developed countries, and $25 \%$ in developing countries [10]. Consequently, this will have a high impact on the quality of care, reflected in increased morbidity and mortality rates.

In the US, hospital patients contract two million infections per year, approximately one infection for every 25 patients [11]. These infections can be life-threatening and difficult to treat. Hand hygiene is considered one of the most simple but important ways to break the chain of infection [12]. Proper hand hygiene before and after each contact with any patient is an important measure to prevent HCRI [9] [11].

There are limited studies regarding HH in Palestine so far. Furthermore, there are no established and organized systems within the Ministry of Health $(\mathrm{MOH})$ in Palestine for registering HCRIs. Health care workers, especially nurses and physicians, have the most physical contact with patients, and thus they are the primary vector for infection transmission within hospitals.

Although HCRI is a major threat to patients' health and safety, it is highly preventable by proper HH [13]. Enhancing and promoting compliance of health care workers with HH is very important to prevent HCRI, and this needs to be based on baseline data by assessing the knowledge, practices and attitudes of Palestinian health care workers. Furthermore, studying and comparing knowledge, practices and attitudes regarding $\mathrm{HH}$ and the results may be used to reform and change the curricula for health sciences, as following the guidelines for proper HH may reduce HCRI by one-third [14]. Assessing the knowledge, practices and attitudes for physicians and nurses may also help in recognizing the factors that affect their compliance with $\mathrm{HH}$, as it is still low [8].

Hand hygiene is an important aspect of the care provided to hospitalized patients [13]. Hand hygiene generally refers to hand washing with water and soap, or by using antiseptic solution or alcohol-based hand rubs [9]. Hand hygiene saves lives, so that it is very important to assess how much information or knowledge physicians and nurses have about $\mathrm{HH}$ [11]. Hand hygiene also is an effective and cost-efficient way to reduce the number of microorganisms, thereby reducing the rate of transfer of microorganisms to hospitalized patients and this will reduce the number of HCRI [15]. Therefore, the aim of this study was to assess the knowledge, practice and attitude of Palestinian physicians and nurses regarding HH in hospitals. More specifically, the objectives were to: (1) assess the levels of compliance, importance of $\mathrm{HH}$, knowledge, practice, and attitudes of $\mathrm{HH}$ among Palestinian physicians and nurses; (2) compare between physicians and nurses regarding knowledge, practice, and attitude of $\mathrm{HH}$; and (3) assess the differences across age groups, gender, and the experience regarding knowledge, practices and attitudes of $\mathrm{HH}$.

\section{Methods}

\subsection{Design and Settings}

A cross-sectional, descriptive design was conducted in the major districts in governmental and private hospitals in the West Bank, Palestine. Data was collected from the participants using Hand Hygiene Questionnaire, which has been demonstrated to be a valid and reliable instrument [16].

\subsection{Sample}

All Palestinian nurses and physicians who worked in governmental and private hospitals represented the population of this study. A convenience sampling technique was used to recruit the participants. The convenience sampling is enrolled the most available individuals to participate in the study of interest [17]. This sampling method is the most common method used across different disciplines [18]. The required sample size was 176 participants (calculated by G power 3.0, power of 0.80 , medium effect size and alpha of 0.05 ). A total of 225 questionnaires were distributed and 200 questionnaires were returned, indicating a high response rate of $89 \%$. The other $11 \%$ did not answer the questionnaire or did not return it. The primary strategy was the drop-and-collect 
technique. This technique involves the hand delivery and subsequent recovery of self-completion questionnaires [19]. This technique had many advantages, including a high response rate and saving time [20]. Additionally, the researcher dealt with the participants face-to-face and directly [19]. By adopting this technique, a clearer picture of the study for the participants was ensured.

\subsection{Ethical Consideration}

Ethical approval was sought and obtained from Birzeit University and permission was granted by the Palestinian $\mathrm{MOH}$ to conduct this study. Finally, permission was taken from ethical committees of each hospital prior to conducting fieldwork. Each package of the questionnaire had a title page with a full explanation about the study aims and expected outcomes, the nature of voluntary participation and the right to withdraw at any time, and the maintenance of confidentiality and privacy. Answering the questionnaires and returning them was considered to indicate consent.

\subsection{Procedure}

After obtaining ethical approval from the relevant ethical committees, potential participants were informed about the study and its related information. All eligible participants who agreed to participate in this study were asked to rate their knowledge, practice and attitudes regarding hand hygiene. Data collection was conducted by four undergraduate nursing students who were trained to maintain interrater reliability regarding data collection. The participants were informed that the data collector would be available in the selected wards to answer any questions, and the researchers' contact information was available to them. Data collection was undertaken from February to May 2015.

\subsection{Measures}

The Hand Hygiene Questionnaire [16] instrument was used to assess the knowledge, practice and attitude regarding hand hygiene. A demographic section was added to the questionnaire to elicit information on gender, discipline, age, type of hospital and experience. Hand hygiene questionnaire consists of three scales: knowledge, practice and attitude. The first scale contains 12 multiple-choice questions to assess hand hygiene knowledge. The practice and attitude scales were assessed via a five-point Likert-type rating system named the Hand Hygiene Practices Inventory (HHPI), which consists of 14items, and the Hand Hygiene Beliefs Scale (HBS), which consists of 24 items. These Likert scales were summated multi-item scales. There were also certain questions regarding the importance of hand hygiene as perceived by the participants, measured subjectively on a scale from 1 to 10, and compliance with hand hygiene question, which was measured by a percentage. For the purposes of this study, some modifications were applied to the questionnaire in a way that did not affect its psychometric properties.

\subsection{Analysis}

Data was analyzed using SPSS version 19. A score of one was given for each correct answer and a score of zero was given for each wrong answer in knowledge score. The higher the score, the more knowledgeable the participant in relation to hand hygiene. Practices and attitudes means were calculated by adding the summated items: the higher the score, the better the practices and attitudes toward hand hygiene. Descriptive analysis was performed to describe the sample in terms of mean, standard deviation and range. Inferential statistics was performed to assess the difference between nurses and physicians, gender and hospitals regarding the compliance, importance of hand hygiene, knowledge, practice and attitude. As there was an increased risk of type 1 error in using consecutive t-tests, a MANOVA was performed to investigate discipline differences in knowledge, practice, attitude, compliance and importance of hand hygiene. Preliminary data screening were conducted. The scores were reasonably normally distributed. Moreover, linear relationships among pairs of dependent variables were noticed, with no extreme outliers, and no multicollinearity. Therefore, MANOVA was run across the analysis. Missing data was assessed and excluded from the analysis.

\section{Results}

\subsection{Sample Characteristics}

As shown in Table 1, a total of 200 subjects participated in this study, most of whom were male $(74.5 \%, \mathrm{n}=$ 
Table 1. Demographic characteristics of the sample.

\begin{tabular}{|c|c|c|c|c|c|c|c|}
\hline Variable & $\mathrm{N}$ & $\%$ & M & SD & $\mathrm{P}_{50}$ & Min & Max \\
\hline Age & - & - & 30.6 & 7.4 & 29 & 20 & 60 \\
\hline Gender: & 149 & 74.5 & & & & & \\
\hline $\begin{array}{c}\text { Male } \\
\text { Female }\end{array}$ & 51 & 25.5 & - & - & - & - & - \\
\hline Job: & 57 & 28.5 & & & & & \\
\hline Physicians & & & - & - & - & - & - \\
\hline Nurses & 143 & 71.5 & & & & & \\
\hline YoE & - & - & 7.3 & 6.8 & 6 & 1 & 36 \\
\hline $\mathrm{CoE}$ & 142 & 71 & & & & & \\
\hline Palestine & & & - & - & - & - & - \\
\hline Others & 58 & 29 & & & & & \\
\hline Hospital & 112 & 56 & & & & & \\
\hline Governmental & & & - & - & - & - & - \\
\hline Private & 88 & 44 & & & & & \\
\hline
\end{tabular}

${ }^{*} \mathrm{~N}=200$; ${ }^{*}$ YoE: Years of Experience, CoE: Country of Education.

149), while a quarter were female $25.5 \%(n=51)$. The mean for age was $30.6 \pm 7.4$. As expected, nurses represented most of the sample $71.5 \%(n=143)$ with $57 \%(n=82)$ had a bachelor's degree in nursing, while physicians represented $28.5 \%(n=57)$ of the sample. The mean for years of experience was $7.3 \pm 6.8$. The majority of the sample graduated from Palestinian universities and schools for both nurses and physicians (71\%, $n=142)$, while $29 \%(n=58)$ graduated from outside universities. Regarding the hospitals, more than half of the sample were governmental employees $(56 \%, n=112)$, while private sector employees represent $44 \%$ of the final sample $(\mathrm{n}=88)$.

\subsection{Knowledge, Practice and Attitude}

Table 2 shows that more than half of the sample reported that they had received training about hand hygiene $(57 \%, n=114)$, compared with $43 \%(n=86)$ who did not get any training. The mean for compliance in hand hygiene for the participants was $80 \pm 14$. The mean for importance of hand hygiene according to the participants' perceptions was $9.2 \pm 1.4$. In general, the sample has a moderate knowledge regarding the hand hygiene $(\mathrm{m}=6$, $\mathrm{SD}=1.7$ ). In relation to practice, the participants had a mean score of $62 \pm 7$. The sample has a better attitude score than practice with a mean of $82.5 \pm 8.8$.

\subsection{Inferential Statistics}

There were no statistically significant differences between nurses and physicians regarding importance of hand hygiene, compliance, knowledge, practice and attitude scores (Table 3). The same results were found when gender was assessed for these differences (Table 4). There was a significant difference between male and female only in practice ( $\mathrm{f}=5.98, p=0.015$ ). Regarding age, it was found that there were no statistically significant differences among age groups across compliance in hand hygiene, importance of hand hygiene, knowledge and practice, while attitude was statistically significant $(f=5.126, p=0.002)$ and post hoc analysis showed that the older the nurses and physicians, the more diligent their practice toward hand hygiene.

In addition, it was found that there were statistically significant differences among groups of years of experience in compliance ( $\mathrm{f}=3.599, p=0.015$ ) and attitude score $(\mathrm{f}=4.683, p=0.003)$. Regarding the hospitals, it was found that there were significant differences between governmental and private hospitals in compliance $(\mathrm{f}=$ 7.092, $p=0.008$ ), in perceived importance of hand hygiene ( $\mathrm{f}=4.596, p=0.033)$ and in practice $(\mathrm{f}=5.034, p=$ 0.027); the private hospitals had higher scores for all of these dimensions (Table 5).

\section{Discussion}

Hand hygiene is one of the most important measures to prevent HCRI. It is easy, simple and quick to implement, 
Table 2. Descriptive characteristics of the knowledge, practice, attitude, compliance and importance of hand hygiene.

\begin{tabular}{|c|c|c|c|c|c|}
\hline Variable & M & $\mathrm{SD}$ & $\mathrm{P}_{50}$ & Min & Max \\
\hline Compliance & 80 & 15 & 80 & 10 & 100 \\
\hline $\mathrm{IoHH}$ & 9.2 & 1.4 & 10 & 4 & 6 \\
\hline Kscore & 6 & 1.7 & 6 & 0 & 10 \\
\hline Pscore & 62 & 7 & 63 & 30 & 70 \\
\hline Ascore & 82.5 & 8.8 & 82 & 54 & 121 \\
\hline
\end{tabular}

"N = 200; "IoHH: Importance of Hand Hygiene, K score: knowledge score, P score: practice score, A score: attitude score.

Table 3. Differences in hand hygiene knowledge, practice, attitude, compliance and importance of hand hygiene scores by discipline $(\mathrm{N}=200)$.

\begin{tabular}{|c|c|c|c|c|}
\hline Variable & Discipline & Mean & $\mathrm{F}$ & $\mathrm{P}$ \\
\hline IoHH & $\begin{array}{l}\text { Physicians } \\
\text { Nurses }\end{array}$ & $\begin{array}{l}9.17 \\
9.18\end{array}$ & 0.001 & 0.98 \\
\hline Compliance & $\begin{array}{l}\text { Physicians } \\
\text { Nurses }\end{array}$ & $\begin{array}{l}77.00 \\
81.00\end{array}$ & 3.210 & 0.07 \\
\hline Kscore & $\begin{array}{l}\text { Physicians } \\
\text { Nurses }\end{array}$ & $\begin{array}{l}6.12 \\
5.91\end{array}$ & 0.572 & 0.45 \\
\hline Pscore & $\begin{array}{l}\text { Physicians } \\
\text { Nurses }\end{array}$ & $\begin{array}{l}62.10 \\
61.86\end{array}$ & 0.036 & 0.84 \\
\hline Ascore & $\begin{array}{c}\text { Physicians } \\
\text { Nurses }\end{array}$ & $\begin{array}{l}83.10 \\
82.26\end{array}$ & 0.339 & 0.56 \\
\hline
\end{tabular}

*N = 200; "IoHH: importance of hand hygiene, K score: knowledge score, P score: practice score, A score: attitude score.

Table 4. Differences in hand hygiene knowledge, practice, attitude, compliance and importance of hand hygiene scores by gender.

\begin{tabular}{|c|c|c|c|c|}
\hline Variable & Discipline & Mean & F & $\mathrm{P}$ \\
\hline IoHH & $\begin{array}{l}\text { Male } \\
\text { Female }\end{array}$ & $\begin{array}{l}9.07 \\
9.50\end{array}$ & 3.65 & 0.06 \\
\hline Compliance & $\begin{array}{c}\text { Male } \\
\text { Female }\end{array}$ & $\begin{array}{l}78.98 \\
83.20\end{array}$ & 3.102 & 0.08 \\
\hline Kscore & $\begin{array}{c}\text { Male } \\
\text { Female }\end{array}$ & $\begin{array}{l}5.90 \\
6.22\end{array}$ & 1.307 & 0.25 \\
\hline Pscore & $\begin{array}{l}\text { Male } \\
\text { Female }\end{array}$ & $\begin{array}{l}61.21 \\
63.98\end{array}$ & 5.984 & 0.015 \\
\hline Ascore & $\begin{array}{l}\text { Male } \\
\text { Female }\end{array}$ & $\begin{array}{l}81.78 \\
84.56\end{array}$ & 3.852 & 0.051 \\
\hline
\end{tabular}

"N = 200; "IoHH: importance of hand hygiene, K score: knowledge score, P score: practice score, A score: attitude score.

Table 5. Differences in hand hygiene knowledge, practice, attitude, compliance and importance of hand hygiene scores by hospitals.

\begin{tabular}{|c|c|c|c|c|}
\hline Variable & Discipline & Mean & $\mathrm{F}$ & $\mathrm{P}$ \\
\hline $\mathrm{IoHH}$ & $\begin{array}{l}\text { Governmental } \\
\text { Private }\end{array}$ & $\begin{array}{l}9.00 \\
9.41\end{array}$ & 4.596 & 0.03 \\
\hline Compliance & $\begin{array}{l}\text { Governmental } \\
\text { Private }\end{array}$ & $\begin{array}{l}77.62 \\
83.16\end{array}$ & 7.092 & 0.00 \\
\hline Kscore & $\begin{array}{l}\text { Governmental } \\
\text { Private }\end{array}$ & $\begin{array}{l}5.92 \\
6.05\end{array}$ & 0.256 & 0.61 \\
\hline Pscore & $\begin{array}{c}\text { Governmental } \\
\text { Private }\end{array}$ & $\begin{array}{l}61.86 \\
62.00\end{array}$ & 5.340 & 0.02 \\
\hline Ascore & $\begin{array}{l}\text { Governmental } \\
\text { Private }\end{array}$ & $\begin{array}{l}82.54 \\
82.43\end{array}$ & 0.008 & 0.93 \\
\hline
\end{tabular}

${ }^{*} \mathrm{~N}=200 ;$; IoHH: importance of hand hygiene, K score: knowledge score, P score: practice score, A score: attitude score. 
but it has an immense impact on HCRI. In the current study, the sample has a moderate knowledge and practice of $\mathrm{HH}$. In general, the sample has a better score on attitude compared to knowledge and practice of $\mathrm{HH}$. These findings are consistent with previous work by [21]. These results are considered positive findings, especially as the study was conducted in a developing country (Palestine). Unexpectedly, compliance with HH was higher than in previous studies as the mean score was $80 \%$, indicating encouraging rates compared with previous research [7].

Nurses and physicians had almost the same rated knowledge, practice and attitude regarding HH. Moreover, compliance and importance of $\mathrm{HH}$ had no significant differences between nurses and physicians. These results contradict the findings of most of the previous studies [16] [21]. Nair et al. [21] examined knowledge, attitude and practice of $\mathrm{HH}$ among medical and nursing students in India. They found that nursing students had more knowledge and compliance in HH than medical students $(p<0.05)$. Similarly, Van de Mortel et al. [16] found that hand hygiene knowledge and practices were significantly higher in nursing students than among medical students.

In contrast, Abd Elaziz and Bakr [22] found that doctors showed a significantly higher compliance than other groups of health care workers. This could be due to that majority of both nurses and physicians graduated from Palestinian nursing and medical schools. This may make more similarities than discrepancies, even though physicians study for two more years than nurses. This could also reflect the knowledge-practice gap in nursing (whereby the theoretical knowledge of nursing students, which is generally superior for $\mathrm{HH}$, gets lost in the translation into practice due to nursing overwork and other practice factors associated with nurses' intense patient interaction).

However, it was found that older participants (with more clinical experience) had better attitudes regarding HH. Conversely, Rajcevic et al. [7] found that health care workers under 40 years old had more knowledge and compliance regarding $\mathrm{HH}$ compared to their older peers. These contradictory results may be related to the differences in the undergraduate curriculum, which may have been reformed to focus more on the prevention of HCRI and HH. It may also be related to the accumulated experience and more courses among Palestinian nurses and physicians. The participants in the private sectors rated better compliance, importance of $\mathrm{HH}$ and practice than governmental sectors. This could be explained by availability of HH facilities and more continuous education regarding $\mathrm{HH}$ in the private sector.

Although the results of the current study showed that moderate knowledge, practice and attitude and high compliance were reported from the participants, future research should focus on overt and covert observational study to detect $\mathrm{HH}$ practice and attitude more closely, to detect more accurate information, as social desirability bias among the participants' answers could be an issue in this study. Also, investigating HH qualitatively is important to assess the limiting factors in attitudes, practices and compliance of HH. Descriptive design and convenience sampling are also limitations in this study. Further research is needed regarding $\mathrm{HH}$ in the clinical settings.

\section{Conclusions}

In conclusion, HH is very important to health care workers to control HCRI. It is important for both physicians and nurses and other healthcare workers to stick to and improve their practice, attitude and knowledge about this issue, which will play an important role in decreasing the cost of care for the patients and hospitals, contributing to shorter hospital stay, reducing mortality and morbidity rates and improving quality of care and of life for patients. This study shows that both Palestinian nurses and physicians have moderate knowledge, practice and attitude regarding $\mathrm{HH}$. Chain of infection is a vicious circle that repeats itself, thus it is very important to break this chain by $\mathrm{HH}$.

This study gives clues for clinical practice in relation to the HH. In spite of moderate results regarding knowledge, practice and attitude of $\mathrm{HH}$, there is a need to improve the clinical daily routines for nurses and the doctors. The absence of significant differences between them in most of the study variables makes equal need for continuous education regarding HH for both Palestinian nurses and physicians. Furthermore, the significant differences between governmental and private hospitals regarding compliance and practice draws attention toward the availability of $\mathrm{HH}$ facilities in the former.

\section{References}

[1] World Health Organization (2012) Hand Hygiene: WHO Highlights Importance of Good Hand Hygiene for Patient 
Safety. http://www.who.int/mediacentre/news/notes/2012/hygiene_20120504/en/

[2] Daugherty, E.L., et al. (2012) Safety Culture and Hand Hygiene: Linking Attitudes to Behavior. Infection Control, 33, 1280-1282. http://dx.doi.org/10.1086/668432

[3] World Health Organization (2009) Patient Safety: WHO Guidelines on Hand Hygiene in Health Care-First Global Patient Safety Challenge, Clean Care Is Safer Care. World Health Organization, Geneva.

[4] Weber, D.J., Anderson, D. and Rutala, W.A. (2013) The Role of the Surface Environment in Healthcare-Associated Infections. Current Opinion in Infectious Diseases, 26, 338-344. http://dx.doi.org/10.1097/QCO.0b013e3283630f04

[5] Ellingson, K., et al. (2014) Strategies to Prevent Healthcare-Associated Infections through Hand Hygiene. Infection Control \& Hospital Epidemiology, 35, 937-960. http://dx.doi.org/10.1086/651677

[6] World Health Organization (2014) Good Hand Hygiene by Health Workers Protects Patients from Drug Resistant Infections. http://www.who.int/mediacentre/news/releases/2014/hand-hygiene/en/

[7] Rajcevic, S., et al. (2012) Knowledge, Habits and Attitudes of Health Care Workers about Hand Hygiene. HealthMed, 6, 1418-1423.

[8] Kendall, A., et al. (2012) Point-of-Care Hand Hygiene: Preventing Infection behind the Curtain. American Journal of Infection Control, 40, S3-S10. http://dx.doi.org/10.1016/j.ajic.2012.02.009

[9] Hillier, M.D. (2015) How to Wash Your Hands Effectively. Nursing Standard, 30, 34-36. http://dx.doi.org/10.7748/ns.30.3.34.e9691

[10] Rao, M.H., et al. (2012) Assessment of Knowledge, Attitude and Practices Pattern of Hand Washing in Some Major Public Sector Hospitals of Pakistan (a Multi-Center Study). Pakistan Journal of Medical Research, 51, 76.

[11] Centers for Disease Control and Prevention (2010) Hand Hygiene Saves Lives.

[12] Gould, C.V., Umscheid, C.A., Agarwal, R.K., Kuntz, G., Pegues, D.A. and Healthcare Infection Control Practices Advisory Committee (HICPAC) (2010) Guideline for Prevention of Catheter-Associated Urinary Tract Infections 2009. Infection Control and Hospital Epidemiology, 31, 319-320. http://dx.doi.org/10.1086/651091

[13] Luby, S.P., Agboatwalla, M., Feikin, D.R., Painter, J., Billhimer, W., Altaf, A. and Hoekstra, R.M. (2005) Effect of Handwashing on Child Health: A Randomised Controlled Trial. The Lancet, 366, 225-233. http://dx.doi.org/10.1016/S0140-6736(05)66912-7

[14] Baker, G., Norton, P.G., Flintoft, V., Blais, R., Brown, A., Cox, J., et al. (2004) The Canadian Adverse Events Study: The Incidence of Adverse Events among Hospital Patients in Canada. Canadian Medical Association Journal, 170, 1678-1686. http://dx.doi.org/10.1503/cmaj.1040498

[15] Lin, H.-L., Yang, L.-Y. and Lai, C.-C. (2014) Factors Associated with Hand Hygiene Compliance among Critical Care Nurses. Infection Control, 35, 329-330. http://dx.doi.org/10.1086/675298

[16] Van De Mortel, T.F., Kermode, S., Progano, T. and Sansoni, J. (2012) A Comparison of the Hand Hygiene Knowledge, Beliefs and Practices of Italian Nursing and Medical Students. Journal of Advanced Nursing, 68, 569-579. http://dx.doi.org/10.1111/j.1365-2648.2011.05758.x

[17] Polit, D.F. and Beck, C.T. (2004) Nursing Research: Principles and Methods. Seventh Edition, Lippincott Williams \& Wilkins, Philadelphia.

[18] Creswell, J.W. (2013) Research Design: Qualitative, Quantitative, and Mixed Methods Approaches. Second Edition, Sage Publications, Thousand Oaks.

[19] Brown, S. (1987) Drop and Collect Surveys: A Neglected Research Technique. Marketing Intelligence and Planning, 5, 19-23. http://dx.doi.org/10.1108/eb045742

[20] Baker, M. (2003) Business and Management Research: How to Complete Your Research Project Successfully. Westburn Publishers Ltd., Helensburgh.

[21] Nair, S.S., Hanumantappa, R., Hiremath, S.G., Siraj, M.A. and Raghunath, P. (2014) Knowledge, Attitude, and Practice of Hand Hygiene among Medical and Nursing Students at a Tertiary Health Care Centre in Raichur, India. ISRN Preventive Medicine, 2014, Article ID: 608927. http://dx.doi.org/10.1155/2014/608927

[22] Abd Elaziz, K. and Bakr, I.M. (2015) Assessment of Knowledge, Attitude and Practice of Hand Washing among Health Care Workers in Ain Shams University Hospitals in Cairo. Journal of Preventive Medicine and Hygiene, 50, $19-25$. 